\title{
Uniform attractors for non-autonomous suspension bridge-type equations
}

\author{
Xuan Wang ${ }^{1,2^{*}}$, Lu Yang ${ }^{2}$ and Qiaozhen $\mathrm{Ma}^{1}$
}

\section{"Correspondence:}

wangxuan@nwnu.edu.cn

${ }^{1}$ College of Mathematics and

Statistics, Northwest Normal

University, Lanzhou, 730070, P.R. China

${ }^{2}$ School of Mathematics and Statistics, Lanzhou University, Lanzhou, 730000, P.R. China

\begin{abstract}
We discuss the long-time dynamical behavior of the non-autonomous suspension bridge-type equation, where the nonlinearity $g(u, t)$ is translation compact and the time-dependent external forces $h(x, t)$ only satisfy Condition $\left(C^{*}\right)$ instead of being translation compact. By applying some new results and the energy estimate technique, the existence of uniform attractors is obtained. The result improves and extends some known results.
\end{abstract}

MSC: 34Q35; 35B40; 35B41

Keywords: non-autonomous suspension bridge equation; uniform Condition (C); uniform attractor

\section{Introduction}

Consider the following equations:

$$
\begin{cases}u_{t t}+u_{x x x x}+\delta u_{t}+k u^{+}=l+\epsilon h(x, t), & \text { in }(0, L) \times \mathbb{R}, \\ u(0, t)=u(L, t)=u_{x x}(0, t)=u_{x x}(L, t)=0, & t \in \mathbb{R} .\end{cases}
$$

Suspension bridge equations (1.1) have been posed as a new problem in the field of nonlinear analysis [1] by Lazer and McKenna in 1990. This model has been derived as follows. In the suspension bridge system, the suspension bridge can be considered as an elastic and unloaded beam with hinged ends. $u(x, t)$ denotes the deflection in the downward direction; $\delta u_{t}$ represents the viscous damping. The restoring force can be modeled owing to the cable with one-sided Hooke's law so that it strongly resists expansion but does not resist compression. The simplest function to model the restoring force of the stays in the suspension bridge can be denoted by a constant $k$ times $u$, the expansion, if $u$ is positive, but zero if $u$ is negative, corresponding to compression; that is, $k u^{+}$, where

$$
u^{+}= \begin{cases}u, & \text { if } u>0 \\ 0, & \text { if } u \leq 0\end{cases}
$$

Besides, the right-hand side of (1.1) also contains two terms: the large positive term $l$ corresponding to gravity, and a small oscillatory forcing term $\epsilon h(x, t)$, possibly aerodynamic in origin, where $\epsilon$ is small.

There are many results for the problem (1.1) ( $c f$. [1-7]), for instance, the existence, multiplicity and properties of the traveling wave solutions, etc.

\section{楚 Springer}

๑2014 Wang et al.; licensee Springer. This is an Open Access article distributed under the terms of the Creative Commons Attribution License (http://creativecommons.org/licenses/by/2.0), which permits unrestricted use, distribution, and reproduction in any medium, provided the original work is properly cited. 
In the study of equations of mathematical physics, the attractor is a proper mathematical concept as regards the depiction of the behavior of the solutions of these equations when time is large or tends to infinity, which describes all the possible limits of solutions. In the past two decades, many authors have proved the existence of an attractor and discussed its properties for various mathematical physics models (e.g., see [8-10] and the references therein). For the long-time behavior of suspension bridge-type equations, for the autonomous case, in $[11,12]$ the authors have discussed long-time behavior of the solutions of the problem on $\mathbb{R}^{2}$ and obtained the existence of global attractors in the space $H_{0}^{2}(\Omega) \times L^{2}(\Omega)$ and $D(A) \times H_{0}^{2}(\Omega)$.

It is well known that, for a model to describe the real world which is affected by many kinds of factors, the corresponding non-autonomous model is more natural and precise than the autonomous one, moreover, it always presents a nonlinear equation but not just a linear one. Therefore, in this paper, we will discuss the following non-autonomous suspension bridge-type equation: Let $\Omega$ be an open bounded subset of $\mathbb{R}^{2}$ with smooth boundary, $\mathbb{R}_{\tau}=[\tau,+\infty]$, and we add the nonlinear forcing term $g(u, t)$ (which is dependent on the deflection $u$ and time $t$ ) to (1.1) and neglect gravity, then we can obtain the following initial-boundary value problem:

$$
\begin{cases}u_{t t}+\Delta^{2} u+\alpha u_{t}+k u^{+}+g(u, t)=h(x, t), & \text { in } \Omega \times \mathbb{R}_{\tau}, \\ u(x, t)=\Delta u(x, t)=0, & \text { on } \partial \Omega \times \mathbb{R}_{\tau}, \\ u(x, \tau)=u_{1}(x), \quad u_{t}(x, \tau)=u_{2}(x), & x \in \Omega,\end{cases}
$$

where $u(x, t)$ is an unknown function, which could represent the deflection of the road bed in the vertical plane; $h(x, t)$ and $g(u, t)$ are time-dependent external forces; $k u^{+}$represents the restoring force, $k$ denotes the spring constant; $\alpha u_{t}$ represents the viscous damping, $\alpha$ is a given positive constant.

To our knowledge, this is the first time for one to consider the non-autonomous dynamics of equation (1.2). At the same time, in mathematics, we only assume that the force term $h(x, t)$ satisfies the so-called Condition $\left(C^{*}\right)$ (introduced in [13]), which is weaker than the assumption of being translation compact (see [8] or Section 2 below).

This paper is organized as follows. At first, in Section 2, we give (recall) some preliminaries, including the notation we will use, the assumption on nonlinearity $g(\cdot, t)$ and some general abstract results for a non-autonomous dynamical system. In Section 3 we prove our main result about the existence of a uniform attractor for the non-autonomous dynamical system generated by the solution of (1.2).

\section{Notation and preliminaries}

With the usual notation, we introduce the spaces $H=L^{2}(\Omega), V=H^{2}(\Omega) \cap H_{0}^{1}(\Omega), D(A)=$ $\left\{u \in H^{2}(\Omega) \cap H_{0}^{1}(\Omega) \mid A u \in L^{2}(\Omega)\right\}$, where $A=\Delta^{2}$. We equip these spaces with an inner product and a norm $\langle\cdot, \cdot\rangle,\|\cdot\|,\langle\cdot, \cdot\rangle_{1},\|\cdot\|_{1}$ and $\langle\cdot, \cdot\rangle_{2},\|\cdot\|_{2}$, respectively,

$$
\begin{aligned}
& \langle u, v\rangle=\int_{\Omega} u(x) v(x) d x, \quad\|u\|^{2}=\int_{\Omega}|u(x)|^{2} d x, \quad \forall u, v \in H ; \\
& \langle u, v\rangle_{1}=\int_{\Omega} \Delta u(x) \Delta v(x) d x, \quad\|u\|_{1}^{2}=\int_{\Omega}|\Delta u(x)|^{2} d x, \quad \forall u, v \in V ; \\
& \langle u, v\rangle_{2}=\int_{\Omega} \Delta^{2} u(x) \Delta^{2} v(x) d x, \quad\|u\|_{2}^{2}=\int_{\Omega}\left|\Delta^{2} u(x)\right|^{2} d x, \quad \forall u, v \in D(A) .
\end{aligned}
$$


Obviously, we have

$$
D(A) \subset V \subset H=H^{*} \subset V^{*}
$$

where $H^{*}, V^{*}$ is the dual space of $H, V$, respectively, the injections are continuous and each space is dense in the following one.

In the following, the assumption on the nonlinearity $g$ is given. Let $g$ be a $C^{1}$ function from $\mathbb{R} \times \mathbb{R}$ to $\mathbb{R}$ and satisfy

$$
\liminf _{|u| \rightarrow \infty} \frac{G(u, s)}{u^{2}} \geq 0
$$

where $G(u, s)=\int_{0}^{u} g(w, s) d w$, and there exists $C_{0}>0$, such that

$$
\liminf _{|u| \rightarrow \infty} \frac{\langle u, g(u, s)\rangle-C_{0} G(u, s)}{u^{2}} \geq 0 .
$$

Suppose that $\gamma$ is an arbitrary positive constant, and

$$
\begin{aligned}
& \left|g_{u}(u, s)\right| \leq C_{1}\left(1+|u|^{\gamma}\right), \quad\left|g_{s}(u, s)\right| \leq C_{1}\left(1+|u|^{\gamma+1}\right), \\
& G_{s}(u, s) \leq \delta^{2} G(u, s)+C_{2}, \quad \forall(u, s) \in \mathbb{R} \times \mathbb{R},
\end{aligned}
$$

where $\delta$ is a sufficiently small constant.

As a consequence of (2.1)-(2.2), if we denote $\mathcal{G}(u, s)=\int_{\Omega} G(u, s) d x$, then there exist two positive constants $K_{1}, K_{2}$ such that

$$
\begin{aligned}
& \mathcal{G}(\varphi, s)+m\|\varphi\|^{2}+K_{1} \geq 0, \\
& \langle\varphi, g(\varphi, s)\rangle-C_{0} \mathcal{G}(\varphi, s)+m\|\varphi\|^{2}+K_{2} \geq 0, \quad \forall(\varphi, s) \in \mathbb{R} \times \mathbb{R},
\end{aligned}
$$

where $m, C_{0}>0$, and we can take $m$ sufficiently small.

By virtue of (2.3), we can get

$$
|g(u, s)| \leq C_{3}\left(1+|u|^{\gamma+1}\right), \quad|G(u, s)| \leq C_{3}\left(1+|u|^{\gamma+2}\right) .
$$

When $A=\Delta^{2}$, the problem (1.2) is equivalent to the following equations in $H$ :

$$
\left\{\begin{array}{l}
u_{t t}+\alpha u_{t}+A u+k u^{+}+g(u, t)=h(x, t), \\
u(\tau)=u_{1}, \quad u_{t}(\tau)=u_{2} .
\end{array}\right.
$$

From the Poincaré inequality, there exists a proper constant $\lambda_{1}>0$, such that

$$
\lambda_{1}\|u\|^{2} \leq\|u\|_{1}^{2}, \quad \forall u \in V
$$

We introduce the Hilbert spaces

$$
\mathcal{E}_{0}=V \times H,
$$


and endow this space with the norm

$$
\|z\|_{\mathcal{E}_{0}}=\left\|\left(u, u_{t}\right)\right\|_{\mathcal{E}_{0}}=\left(\frac{1}{2}\left(\|u\|_{1}^{2}+\left\|u_{t}\right\|^{2}\right)\right)^{\frac{1}{2}} .
$$

To prove the existence of uniform attractors corresponding to (2.8), we also need the following abstract results (e.g., see [8]).

Let $E$ be a Banach space, and let a two-parameter family of mappings $\{U(t, \tau)\}=$ $\{U(t, \tau) \mid t \geq \tau, \tau \in \mathbb{R}\}$ on $E$ :

$$
U(t, \tau): E \rightarrow E, \quad t \geq \tau, \tau \in \mathbb{R} .
$$

Definition 2.1 ([8]) Let $\Sigma$ be a parameter set. $\left\{U_{\sigma}(t, \tau) \mid t \geq \tau, \tau \in \mathbb{R}\right\}, \sigma \in \Sigma$ is said to be a family of processes in Banach space $E$, if for each $\sigma \in \Sigma,\left\{U_{\sigma}(t, \tau)\right\}$ is a process; that is, the two-parameter family of mappings $\left\{U_{\sigma}(t, \tau)\right\}$ from $E$ to $E$ satisfy

$$
\begin{aligned}
& U_{\sigma}(t, s) \circ U_{\sigma}(s, \tau)=U_{\sigma}(t, \tau), \quad \forall t \geq s \geq \tau, \tau \in \mathbb{R}, \\
& U_{\sigma}(\tau, \tau)=I \text { is the identity operator, } \quad \tau \in \mathbb{R},
\end{aligned}
$$

where $\Sigma$ is called the symbol space and $\sigma \in \Sigma$ is the symbol.

Note that the following translation identity is valid for a general family of processes $\left\{U_{\sigma}(t, \tau)\right\}, \sigma \in \Sigma$, if a problem has unique solvability and for the translation semigroup $\{T(l) \mid l \geq 0\}$ satisfying $T(l) \Sigma=\Sigma$ :

$$
U_{\sigma}(t+l, \tau+l)=U_{T(l) \sigma}(t, \tau), \quad \forall \sigma \in \Sigma, t \geq \tau, \tau \in \mathbb{R}, l \geq 0 .
$$

A set $B_{0} \subset E$ is said to be a uniformly (w.r.t. $\sigma \in \Sigma$ ) absorbing set for the family of processes $\left\{U_{\sigma}(t, \tau)\right\}, \sigma \in \Sigma$ if for any $\tau \in \mathbb{R}$ and $B \in \mathcal{B}(E)$, there exists $t_{0}=t_{0}(\tau, B) \geq \tau$ such that $\bigcup_{\sigma \in \Sigma} U_{\sigma}(t, \tau) \subseteq B_{0}$ for all $t \geq t_{0}$. A set $Y \subset E$ is said to be uniformly (w.r.t. $\sigma \in \Sigma$ ) attracting for the family of processes $\left\{U_{\sigma}(t, \tau)\right\}, \sigma \in \Sigma$, if for any fixed $\tau \in \mathbb{R}$ and every $B \in \mathcal{B}(E)$,

$$
\lim _{t \rightarrow \infty}\left(\sup _{\sigma \in \Sigma} \operatorname{dist}_{E}\left(U_{\sigma}(t, \tau) B, Y\right)\right)=0
$$

Definition 2.2 ([8]) A closed set $A_{\Sigma} \subset E$ is said to be the uniform (w.r.t. $\sigma \in \Sigma$ ) attractor of the family of processes $\left\{U_{\sigma}(t, \tau)\right\}, \sigma \in \Sigma$ if it is uniformly (w.r.t. $\sigma \in \Sigma$ ) attracting (attracting property) and contained in any closed uniformly (w.r.t. $\sigma \in \Sigma$ ) attracting set $A^{\prime}$ of the family of processes $\left\{U_{\sigma}(t, \tau)\right\}, \sigma \in \Sigma: A_{\Sigma} \subseteq A^{\prime}$ (minimality property).

Now we recall the results in [14].

Definition 2.3 ([14]) A family of processes $\left\{U_{\sigma}(t, \tau)\right\}, \sigma \in \Sigma$, is said to be satisfying the uniform (w.r.t. $\sigma \in \Sigma$ ) Condition $(C)$ if for any fixed $\tau \in \mathbb{R}, B \in \mathcal{B}(E)$ and $\epsilon>0$, there exist a $t_{0}=t_{0}(\tau, B, \epsilon) \geq \tau$ and a finite dimensional subspace $E_{m}$ of $E$ such that 
(i) $P_{m}\left(\bigcup_{\sigma \in \Sigma} \bigcup_{t \geq t_{0}} U_{\sigma}(t, \tau) B\right)$ is bounded; and

(ii) $\left\|\left(I-P_{m}\right)\left(\bigcup_{\sigma \in \Sigma} \bigcup_{t \geq t_{0}} U_{\sigma}(t, \tau) x\right)\right\|_{E} \leq \epsilon, \forall x \in B$,

where $\operatorname{dim} E_{m}=m$ and $P_{m}: E \rightarrow E_{m}$ is abounded projector.

Theorem 2.4 ([14]) Let $\Sigma$ be a complete metric space, and let $\{T(t)\}$ be a continuous invariant $T(t) \Sigma=\Sigma$ semigroup on $\Sigma$ satisfying the translation identity. A family of processes $\left\{U_{\sigma}(t, \tau)\right\}, \sigma \in \Sigma$, possess a compact uniform (w.r.t. $\left.\sigma \in \Sigma\right)$ attractor $A_{\Sigma}$ in $E$ satisfying

$$
A_{\Sigma}=\omega_{0, \Sigma}\left(B_{0}\right)=\omega_{\tau, \Sigma}\left(B_{0}\right), \quad \forall t \in \mathbb{R}
$$

if it

(i) has a bounded uniformly (w.r.t. $\sigma \in \Sigma$ ) absorbing set $B_{0}$; and

(ii) satisfies uniform (w.r.t. $\sigma \in \Sigma)$ Condition $(C)$,

where $\omega_{\tau, \Sigma}\left(B_{0}\right)=\bigcap_{t \geq \tau}\left[\bigcup_{\sigma \in \Sigma} \bigcup_{s \geq t} U_{\sigma}(s, t) B_{0}\right]$. Moreover, if $E$ is a uniformly convex Banach space, then the converse is true.

Let $X$ be a Banach space. Consider the space $L_{\text {loc }}^{2}(\mathbb{R} ; X)$ of functions $\phi(s), s \in \mathbb{R}$ with values in $X$ that are 2-power integrable in the Bochner sense. $L_{c}^{2}(\mathbb{R} ; X)$ is a set of all translation compact functions in $L_{\text {loc }}^{2}(\mathbb{R} ; X), L_{b}^{2}(\mathbb{R} ; X)$ is the set of all translation bound functions in $L_{\text {loc }}^{2}(\mathbb{R} ; X)$.

In [13], the authors have introduced a new class of functions which are translation bounded but not translation compact. In Section 3, let the forcing term $h(x, t)$ satisfy Condition $\left(C^{*}\right)$; we can prove the existence of compact uniform (w.r.t. $\sigma \in \mathcal{H}\left(\sigma_{0}\right), \sigma_{0}(s)=$ $\left.\left(g_{0}(u, s), h(x, s)\right)\right)$ attractor for a non-autonomous suspension bridge equation in $\mathcal{E}_{0}$.

Definition 2.5 ([13]) Let $X$ be a Banach space. A function $f \in L_{b}^{2}(\mathbb{R} ; X)$ is said to satisfy Condition $\left(C^{*}\right)$ if, for any $\epsilon>0$, there exists a finite dimensional subspace $X_{1}$ of $X$ such that

$$
\sup _{t \in \mathbb{R}} \int_{t}^{t+1}\left\|\left(I-P_{m}\right) f(s)\right\|_{X}^{2} d s<\epsilon
$$

where $P_{m}: X \rightarrow X_{1}$ is the canonical projector.

Denote by $L_{c^{*}}^{2}(\mathbb{R} ; X)$ the set of all functions satisfying Condition $\left(C^{*}\right)$. From [13], we can see that $L_{c}^{2}(\mathbb{R} ; X) \subset L_{c^{*}}^{2}(\mathbb{R} ; X) \subset L_{b}^{2}(\mathbb{R} ; X)$.

Remark 2.6 In fact, the function satisfying Condition $\left(C^{*}\right)$ implies the dissipative property in some sense, and Condition $\left(C^{*}\right)$ is very natural in view of the compact condition, and the uniform Condition $(C)$.

Lemma 2.7 ([13]) If $\in L_{c^{*}}^{2}(\mathbb{R} ; X)$, then for any $\epsilon>0$ and $\tau \in \mathbb{R}$ we have

$$
\sup _{t \geq \tau} \int_{\tau}^{t} e^{-\delta(t-s)}\left\|\left(I-P_{m}\right) f(s)\right\|_{X}^{2} d s \leq \epsilon
$$

where $P_{m}: X \rightarrow X_{1}$ is the canonical projector and $\delta$ is a positive constant. 
In order to define the family of processes of the equations (2.8), we also need the following results:

Proposition 2.8 ([8]) If $X$ is reflexive separable, then

(i) for all $h_{1} \in \mathcal{H}\left(h_{0}\right),\left\|h_{1}\right\|_{L_{b}^{2}(\mathbb{R} ; X)} \leq\left\|h_{0}\right\|_{L_{b}^{2}(\mathbb{R} ; X)}$;

(ii) the translation group $\{T(t)\}$ is weakly continuous on $\mathcal{H}\left(h_{0}\right)$;

(iii) $T(t) \mathcal{H}\left(h_{0}\right)=\mathcal{H}\left(h_{0}\right)$ for all $t \in \mathbb{R}^{+}$.

Proposition 2.9 ([8]) Let $g_{0}(s) \in L_{c}^{2}(\mathbb{R} ; X)$, then

(i) for all $g_{1} \in \mathcal{H}\left(g_{0}\right), g_{1} \in L_{c}^{2}(\mathbb{R} ; X)$, and the set $\mathcal{H}\left(g_{0}\right)$ is bound in $L_{b}^{2}(\mathbb{R} ; X)$;

(ii) the translation group $\{T(t)\}$ is continuous on $\mathcal{H}\left(g_{0}\right)$ with the topology of $L_{\mathrm{loc}}^{2}(\mathbb{R}, X)$;

(iii) $T(t) \mathcal{H}\left(g_{0}\right)=\mathcal{H}\left(g_{0}\right)$ for all $t \in \mathbb{R}^{+}$.

\section{Uniform attractors in $\mathcal{E}_{0}$}

To describe the asymptotic behavior of the solutions of our system, we set $h_{0} \in L_{c^{*}}^{2}\left(\mathbb{R}_{\tau}\right.$; $H) \subset L_{b}^{2}\left(\mathbb{R}_{\tau} ; H\right)$ and $\mathcal{H}\left(h_{0}\right)=\left[h_{0}(x, s+h) \mid h \in \mathbb{R}\right]_{L_{\mathrm{loc}}^{2, w}\left(\mathbb{R}_{\tau} ; H\right)}$, where [ ] denotes the closure of a set in topological space $L_{\text {loc }}^{2, w}\left(\mathbb{R}_{\tau} ; H\right)$. If $h \in \mathcal{H}\left(h_{0}\right)$, then $h \in L_{b}^{2}\left(\mathbb{R}_{\tau} ; H\right)$; this is to be

$$
\sup _{t \geq \tau} \int_{t}^{t+1}\|h(x, s)\| d s<\infty
$$

where $\|\cdot\|$ denotes the norm in $H$.

\subsection{Existence and uniqueness of solutions}

At first, we give the concept of solutions for the initial-boundary value problem (2.8).

Definition 3.1 Set $I=[\tau, T]$, for $T>\tau \geq 0$. We suppose that $k>0, h \in L_{b}^{2}\left(\mathbb{R}_{\tau} ; H\right), g \in$ $C^{1}(\mathbb{R} \times \mathbb{R} ; \mathbb{R})$ satisfying (2.1)-(2.4) and $g(0,0)=0$. The function $z=\left(u, u_{t}\right) \in C\left(I ; \mathcal{E}_{0}\right)$ is said to be a weak solution to the problem (2.8) in the time interval $I$, with initial data $z(\tau)=z_{\tau}=\left(u_{1}, u_{2}\right) \in \mathcal{E}_{0}$, provided

$$
\left\langle u_{t t}, \bar{v}\right\rangle+\alpha\left\langle u_{t}, \bar{v}\right\rangle+\int_{\Omega} \Delta u \Delta \bar{v} d x+\int_{\Omega} g(u, t) \bar{v} d x+k\left\langle u^{+}, \bar{v}\right\rangle=\int_{\Omega} h(x, t) \bar{v} d x
$$

for all $\bar{v} \in V$ and a.e. $t \in I$.

Then, by using of the methods in [15] (Galerkin approximation method), we get the following result as regards the existence and uniqueness of solutions:

Theorem 3.2 (Existence and uniqueness of solutions) Define $I=[\tau, T], \forall T>\tau$. Let $k>0$, $h \in L_{b}^{2}\left(\mathbb{R}_{\tau} ; H\right), g \in C^{1}(\mathbb{R} \times \mathbb{R} ; \mathbb{R})$ satisfying (2.1)-(2.4). Then for any given $z_{\tau} \in \mathcal{E}_{0}$, there is a unique solution $z=\left(u, u_{t}\right)$ for the problem (2.8) in $\mathcal{E}_{0}$. Furthermore, for $i=1,2$, let $\left\{z_{\tau}^{i}, h_{i}\right\}$ $\left(z_{\tau}^{i} \in \mathcal{E}_{0}\right.$ and $\left.h_{i} \in L_{b}^{2}(\mathbb{R} ; H)\right)$ be two initial conditions, and denote by $z_{i}$ the corresponding solutions to the problem (2.8). Then the estimates hold as follows: for all $\tau \leq t \leq T+\tau$,

$$
\left\|z_{1}(t)-z_{2}(t)\right\|_{\mathcal{E}_{0}}^{2} \leq Q\left(\left\|z_{\tau}^{i}\right\|_{\mathcal{E}_{0}}, T\right)\left(\left\|z_{\tau}^{1}-z_{\tau}^{2}\right\|_{\mathcal{E}_{0}}^{2}+\left\|g_{1}-g_{2}\right\|_{L_{b}^{2}(\mathbb{R} ; H)}^{2}\right) .
$$


Thus, (2.8) will be written as an evolutionary system, introduced as $z(t)=\left(u(t), u_{t}(t)\right)$ and $z_{\tau}=z(\tau)=\left(u_{1}, u_{2}\right)$ for brevity, as $\|z\|_{\mathcal{E}_{0}}^{2}=\frac{1}{2}\left(\|u\|_{1}^{2}+\left\|u_{t}\right\|^{2}\right)$, the system (2.8) can be written in the operator form

$$
\partial_{t} z=A_{\sigma(t)}(z),\left.\quad z\right|_{t=\tau}=z_{\tau},
$$

where $\sigma(s)=(g(u, s), h(x, s))$ is the symbol of (3.3). If $z_{\tau} \in \mathcal{E}_{0}$, then the problem (3.3) has a unique solution $z(t) \in C\left(\mathbb{R}_{\tau}, \mathcal{E}_{0}\right)$. This implies that the process $\left\{U_{\sigma}(t, \tau)\right\}$ given by the formula $U_{\sigma}(t, \tau) z_{\tau}=z(t)$ is defined in $\mathcal{E}_{0}$.

Now we define the symbol space. A fixed symbol $\sigma_{0}(s)=\left(g_{0}(u, s), h_{0}(x, s)\right)$ can be given, where $h_{0}(x, s)$ is in $L_{c^{*}}^{2}\left(\mathbb{R}_{\tau} ; H\right)$, the function $g_{0}(u, s) \in L_{c}^{2}\left(\mathbb{R}_{\tau} ; \mathcal{M}\right)$ satisfying (2.1)-(2.4), and $\mathcal{M}$ is a Banach space,

$$
\mathcal{M}=\left\{g \in C(\mathbb{R} \times \mathbb{R}, \mathbb{R}) \mid \frac{|g(u)|+\left|g_{s}(u)\right|}{|u|^{\gamma+1}+1}+\frac{\left|g_{u}(u)\right|}{|u|^{\gamma}+1}<\infty\right\},
$$

endowed with the following norm:

$$
\|g\|_{\mathcal{M}}=\sup _{u \in \mathbb{R}}\left\{\frac{|g(u)|+\left|g_{s}(u)\right|}{|u|^{\gamma+1}+1}+\frac{\left|g_{u}(u)\right|}{|u|^{\gamma}+1}\right\} .
$$

Obviously, the function $\sigma_{0}(s)=\left(g_{0}(u, s), h_{0}(x, s)\right)$ is in $L_{c}^{2}\left(\mathbb{R}_{\tau} ; \mathcal{M}\right) \times L_{c^{*}}^{2}\left(\mathbb{R}_{\tau} ; H\right)$. We define $\mathcal{H}\left(\sigma_{0}\right)=\mathcal{H}\left(g_{0}\right) \times \mathcal{H}\left(h_{0}\right)=\left[g_{0}(u, s+l) \mid l \in \mathbb{R}\right]_{L_{\mathrm{loc}}^{2, w}\left(\mathbb{R}_{\tau} ; \mathcal{M}\right)} \times\left[h_{0}(x, s+l) \mid l \in \mathbb{R}\right]_{L_{\mathrm{loc}}^{2, w}\left(\mathbb{R}_{\tau} ; H\right)}$, where [] denotes the closure of a set in topological space $L_{\text {loc }}^{2, w}\left(\mathbb{R}_{\tau} ; \mathcal{M}\right)$ (or $\left.L_{\text {loc }}^{2, w}\left(\mathbb{R}_{\tau} ; H\right)\right)$. So, if $(g, h) \in$ $\mathcal{H}\left(\sigma_{0}\right)$, then $g(u, t)$ and $h(x, t)$ all satisfy Condition $\left(C^{*}\right)$.

Applying Proposition 2.8, Proposition 2.9, and Theorem 3.2, we can easily know that the family of processes $\left\{U_{\sigma}(t, \tau)\right\}: \mathcal{E}_{0} \rightarrow \mathcal{E}_{0}, \sigma \in \mathcal{H}\left(\sigma_{0}\right), t \geq \tau$, are defined. Furthermore, the translation semigroup $\left\{T(l) \mid l \in \mathbb{R}^{+}\right\}$satisfies $\forall l \in \mathbb{R}^{+}, T(l) \mathcal{H}\left(\sigma_{0}\right)=\mathcal{H}\left(\sigma_{0}\right)$, and the following translation identity:

$$
U_{\sigma}(t+l, \tau+l)=U_{T(l) \sigma}(t, \tau), \quad \forall \sigma \in \mathcal{H}\left(\sigma_{0}\right), \text { for } t \geq \tau \geq 0, l \geq 0
$$

holds.

Then for any $\sigma \in \mathcal{H}\left(\sigma_{0}\right)$, the problem (3.3) with $\sigma$ instead of $\sigma_{0}$ possesses a corresponding process $\left\{U_{\sigma}(t, \tau)\right\}$ acting on $\mathcal{E}_{0}$.

Consequently, for each $\sigma \in \mathcal{H}\left(\sigma_{0}\right), \sigma_{0}(s)=\left(g_{0}(u, s), h_{0}(x, s)\right)$ (here $h_{0}(x, s) \in L_{c^{*}}^{2}\left(\mathbb{R}_{\tau} ; H\right)$, $g_{0}(u, s) \in L_{c}^{2}\left(\mathbb{R}_{\tau} ; \mathcal{M}\right)$ satisfying $\left.(2.1)-(2.4)\right)$, we can define a process

$$
\begin{aligned}
& U_{\sigma}(t, \tau): \mathcal{E}_{0} \rightarrow \mathcal{E}_{0}, \\
& z_{\tau}=\left(u_{1}, u_{2}\right) \rightarrow\left(u(t), u_{t}(t)\right)=U_{\sigma}(t, \tau) z_{\tau},
\end{aligned}
$$

and $U_{\sigma}(t, \tau), \sigma \in \mathcal{H}\left(\sigma_{0}\right)$, is a family of processes on $\mathcal{E}_{0}$.

\subsection{Bounded uniformly absorbing set}

Before we show the existence of bounded uniformly absorbing set, we firstly make a prior estimate of solutions for equations (2.8) in $\mathcal{E}_{0}$. 
Lemma 3.3 Assume that $z(t)$ is a solution of (2.8) with initial data $z_{0} \in B$. If the nonlinearity $g(u, t)$ satisfies (2.1)-(2.4), $h_{0} \in L_{c^{*}}^{2}\left(\mathbb{R}_{\tau} ; H\right), h \in \mathcal{H}\left(h_{0}\right), k>0$, then there is a positive constant $\mu_{0}$ such that for any bounded (in $\left.\mathcal{E}_{0}\right)$ subset $B$, there exists $t_{0}=t_{0}\left(\|B\|_{\mathcal{E}_{0}}\right)$ such that

$$
\|z(t)\|_{\mathcal{E}_{0}}^{2}=\frac{1}{2}\left(\|u\|_{1}^{2}+\left\|u_{t}\right\|^{2}\right) \leq \mu_{0}^{2}, \quad t \geq t_{0}=t_{0}\left(\|B\|_{\mathcal{E}_{0}}\right)
$$

Proof Now we will prove $z=\left(u, u_{t}\right)$ to be bounded in $\mathcal{E}_{0}=V \times H$.

We assume that $\varrho$ is positive and satisfies

$$
0<\varrho(\alpha-\varrho)<\lambda_{1}
$$

Multiplying (2.8) by $v(t)=u_{t}(t)+\varrho u(t)$ and integrating over $\Omega$, we have

$$
\begin{aligned}
& \frac{1}{2} \frac{d}{d t}\left(\|v\|^{2}+\|u\|_{1}^{2}\right)+\varrho\|u\|_{1}^{2}+(\alpha-\varrho)\|v\|^{2}-\varrho(\alpha-\varrho)\langle u, v\rangle \\
& \quad+k\left\langle u^{+}, v\right\rangle+\langle g(u, t), v\rangle=\langle h(t), v\rangle .
\end{aligned}
$$

We easily see that

$$
\begin{aligned}
& \varrho(\alpha-\varrho)\langle u, v\rangle \leq(\alpha-\varrho) \frac{\|v\|^{2}}{4}+(\alpha-\varrho) \varrho^{2}\|u\|^{2}, \\
& \langle h(t), v\rangle \leq(\alpha-\varrho) \frac{\|v\|^{2}}{4}+\frac{\|h(t)\|^{2}}{\alpha-\varrho} .
\end{aligned}
$$

Then, substituting (3.7)-(3.8) into (3.6), we obtain

$$
\begin{aligned}
& \frac{d}{d t}\left(\|v\|^{2}+\|u\|_{1}^{2}\right)+2 \varrho\|u\|_{1}^{2}+(\alpha-\varrho)\|v\|^{2}-2 \varrho^{2}(\alpha-\varrho)\|u\|^{2} \\
& \quad+2 k\left\langle u^{+}, v\right\rangle+2\langle g, v\rangle \leq 2 \frac{\|h(t)\|^{2}}{\alpha-\varrho} .
\end{aligned}
$$

In view of (2.4) and (2.6), we know

$$
\begin{aligned}
\langle g, v\rangle= & \left\langle g, u_{t}+\varrho u\right\rangle \\
= & \frac{d}{d t} \int_{\Omega} G(u(x, t), t) d x+\varrho|g(u, t), u\rangle-\int_{\Omega} G_{s}(u(x, t), t) d x \\
= & \frac{d}{d t} \int_{\Omega} G(u(x, t), t) d x+\varrho \int_{\Omega} g(u(x, t), t) u(x, t) d x \\
& -\varrho C_{0} \int_{\Omega} G(u(x, t), t) d x+\varrho C_{0} \int_{\Omega} G(u(x, t), t) d x \\
& -\int_{\Omega} G_{s}(u(x, t), t) d x \\
\geq & \frac{d}{d t} \mathcal{G}(u(x, t), t)+\varrho C_{0} \mathcal{G}(u(x, t), t)-\varrho\left(m\|u\|^{2}+K_{2}\right) \\
& -\delta^{2} \mathcal{G}(u(x, t), t)-C_{2}|\Omega|
\end{aligned}
$$


and

$$
k\left\langle u^{+}, v\right\rangle=\frac{1}{2} \frac{d}{d t} k\left\|u^{+}\right\|^{2}+\varrho k\left\|u^{+}\right\|^{2} .
$$

Consequently,

$$
\begin{aligned}
\frac{d}{d t}( & \left.\|v\|^{2}+\|u\|_{1}^{2}+k\left\|u^{+}\right\|^{2}+2 \mathcal{G}(u(x, t), t)\right)+(\alpha-\varrho)\|v\|^{2} \\
& +2 \frac{\varrho}{\lambda_{1}}\left(\lambda_{1}-\varrho(\alpha-\varrho)-m\right)\|u\|_{1}^{2}+2 \varrho k\left\|u^{+}\right\|^{2} \\
& +\left(\varrho C_{0}-\delta^{2}\right) 2 \mathcal{G}(u(x, t), t) \\
\leq & 2 \frac{\|h(t)\|^{2}}{\alpha-\varrho}+2\left(\varrho K_{2}+C_{2}|\Omega|\right) .
\end{aligned}
$$

We introduce the functional as follows:

$$
y(t)=\|v\|^{2}+\|u\|_{1}^{2}+k\left\|u^{+}\right\|^{2}+2 \mathcal{G}(u(x, t), t)+2 K_{1}, \quad \text { for } t \geq \tau .
$$

Setting $\beta=\min \left\{\alpha-\varrho, 2 \varrho \lambda_{1}^{-1}\left(\lambda_{1}-\varrho(\alpha-\varrho)-m\right), 2 \varrho, \varrho C_{0}-\delta^{2}\right\}$, we choose proper positive constants $m$ and $\delta$, such that

$$
m<\lambda_{1}-\varrho(\alpha-\varrho), \quad \delta^{2}<\varrho C_{0}
$$

hold, then $\beta>0$.

We define $m_{h}(t)=\|h(t)\|^{2}$, then

$$
\frac{d}{d t} y(t)+\beta y(t) \leq C_{4}+C_{5} m_{h}(t),
$$

where $C_{4}=2\left(\varrho K_{2}+C_{2}|\Omega|\right)+2 \beta K_{1}, C_{5}=2(\alpha-\varrho)^{-1}$.

Analogous to the proof of Lemma 2.1.3 in [8], we can estimate the integral and obtain

$$
\begin{aligned}
y(t) \leq & y(\tau) e^{-\beta(t-\tau)}+C_{4} \beta^{-1}\left(1-e^{-\beta t}\right)+C_{5} \int_{0}^{t} m_{h}(s) e^{-\beta(t-s)} d s \\
\leq & y(\tau) e^{-\beta(t-\tau)}+C_{4} \beta^{-1}\left(1-e^{-\beta t}\right)+C_{5} \int_{t-1}^{t} m_{h}(s) e^{-\beta(t-s)} d s \\
& \quad+C_{5} \int_{t-2}^{t-1} m_{h}(s) e^{-\beta(t-s)} d s+\cdots \\
\leq & y(\tau) e^{-\beta(t-\tau)}+C_{4} \beta^{-1}\left(1-e^{-\beta t}\right)+C_{5} \int_{t-1}^{t} m_{h}(s) d s \\
& +C_{5} e^{-\beta} \int_{t-2}^{t-1} m_{h}(s) d s+C_{5} e^{-2 \beta} \int_{t-3}^{t-2} m_{h}(s) d s+\cdots \\
\leq & y(\tau) e^{-\beta(t-\tau)}+C_{4} \beta^{-1}\left(1-e^{-\beta t}\right)+C_{5} m\left(1+e^{-\beta}+e^{-2 \beta}+\cdots\right) \\
\leq & y(\tau) e^{-\beta(t-\tau)}+C_{4} \beta^{-1}\left(1-e^{-\beta t}\right)+C_{5} m\left(1+\beta^{-1}\right) \\
\leq & y(\tau) e^{-\beta(t-\tau)}+C_{4} \beta^{-1}+C_{5} m\left(1+\beta^{-1}\right), \quad \text { for } t \geq \tau,
\end{aligned}
$$

where $m=\sup _{t \geq \tau} \int_{t}^{t+1} m_{h}(s) d s$. 
By virtue of (2.3), we get

$$
2 \mathcal{G}(u, t) \geq-2 m\|u\|^{2}-2 K_{1} \geq-2 m \lambda_{1}^{-1}\|u\|_{1}^{2}-2 K_{1}
$$

Choosing $m \leq \lambda_{1} / 4$, we obtain from (3.13)

$$
\begin{aligned}
y(t) & =\|u\|_{1}^{2}+\left\|u_{t}+\varrho u\right\|^{2}+k\left\|u^{+}\right\|^{2}+2 \mathcal{G}(u, t)+2 K_{1} \\
& \geq \frac{1}{2}\|u\|_{1}^{2}+\left\|u_{t}+\varrho\right\|^{2}+k\left\|u^{+}\right\|^{2} \\
& \geq\|z(t)\|_{\mathcal{E}_{0}}^{2} .
\end{aligned}
$$

In consideration of (2.7) and $0<\gamma<\infty$, we see

$$
2 \mathcal{G}\left(u_{\tau}(x), \tau\right) \leq 2 C_{3} \int_{\Omega}\left(\left|u_{\tau}(x)\right|^{\gamma+2}+1\right) d x \leq C_{6}\left(\left\|u_{\tau}\right\|_{1}^{\gamma+2}+1\right)
$$

and

$$
\begin{aligned}
y(\tau) & =\|u(\tau)\|_{1}^{2}+\left\|u_{t}(\tau)+\varrho u(\tau)\right\|^{2}+k\left\|(u(\tau))^{+}\right\|^{2}+2 \mathcal{G}(u(\tau), \tau)+2 K_{1} \\
& \leq C_{7}\left(\|z(\tau)\|_{\mathcal{E}_{0}}^{\gamma+2}+1\right) .
\end{aligned}
$$

Combining (3.16), (3.17), and (3.19), we deduce that

$$
\begin{aligned}
\|z(t)\|_{\mathcal{E}_{0}}^{2} & \leq y(\tau) e^{-\beta(t-\tau)}+C_{4} \beta^{-1}+C_{5} m\left(1+\beta^{-1}\right) \\
& \leq C_{7}\left(\|z(\tau)\|_{\mathcal{E}_{0}}^{\gamma+2}+1\right) e^{-\beta(t-\tau)}+C_{4} \beta^{-1}+C_{5} m\left(1+\beta^{-1}\right) \\
& \leq C_{7}\|z(\tau)\|_{\mathcal{E}_{0}}^{\gamma+2} e^{-\beta(t-\tau)}+C_{8}, \quad t \geq \tau .
\end{aligned}
$$

Assuming that $\|z(\tau)\|_{\mathcal{E}_{0}}^{2} \leq R$, as $t \geq t_{0}=t_{0}\left(\|B\|_{\mathcal{E}_{0}}\right)$, we have

$$
\|z(t)\|_{\mathcal{E}_{0}} \leq \mu_{0}
$$

We thus complete the proof.

And then, combining Theorem 3.2 with Lemma 3.3, we get the result as follows.

Theorem 3.4 (Bounded uniformly absorbing set) Presume that $g_{0} \in L_{c}^{2}\left(\mathbb{R}_{\tau} ; M\right)$ and $h_{0} \in$ $L_{c^{*}}^{2}\left(\mathbb{R}_{\tau} ; H\right)$. Let $g \in \mathcal{H}\left(g_{0}\right)$ satisfy $(2.1)-(2.4), h \in \mathcal{H}\left(h_{0}\right)$, and $\left\{U_{\sigma}(t, \tau)\right\}, \sigma \in \mathcal{H}\left(\sigma_{0}\right)=\mathcal{H}\left(g_{0}\right) \times$ $\mathcal{H}\left(h_{0}\right)$ be the family of processes corresponding to $(2.8)$ in $\mathcal{E}_{0}$, then $\left\{U_{\sigma}(t, \tau)\right\}$ has a uniformly (w.r.t. $\left.\sigma \in \mathcal{H}\left(\sigma_{0}\right)\right)$ absorbing set $B_{0}=B_{\mathcal{E}_{0}}\left(0, \mu_{0}\right)$ in $\mathcal{E}_{0}$. That is, for any bounded subset $B \subset$ $\mathcal{E}_{0}$, there exists $t_{0}=t_{0}\left(\|B\|_{\mathcal{E}_{0}}\right)$ such that

$$
\bigcup_{\sigma \in \mathcal{H}\left(\sigma_{0}\right)} U_{\sigma}(t, \tau) B \subset B_{0}, \quad \text { for all } t \geq t_{0} .
$$




\subsection{The existence of uniform attractor}

We will show the existence of uniform attractor to the problem (2.8) in $\mathcal{E}_{0}$.

Theorem 3.5 (Uniform attractor) Let $\left\{U_{\sigma}(t, \tau)\right\}$ be the family of processes corresponding to the problem (2.8). If $g_{0} \in L_{c}^{2}\left(\mathbb{R}_{\tau} ; \mathcal{M}\right)$ satisfying (2.1), (2.2), (2.5), and (2.6), $h_{0} \in L_{c^{*}}^{2}\left(\mathbb{R}_{\tau} ; H\right)$, and $\sigma_{0}=\left(g_{0}, h_{0}\right)$, then $\left\{U_{\sigma}(t, \tau)\right\}$ possesses a compact uniform (w.r.t. $\left.\sigma \in \mathcal{H}\left(\sigma_{0}\right)\right)$ attractor $\mathcal{A}_{\mathcal{H}\left(\sigma_{0}\right)}$ in $\mathcal{E}_{0}$, which attracts any bounded set in $\mathcal{E}_{0}$ with $\|\cdot\|_{\mathcal{E}_{0}}$, satisfying

$$
\mathcal{A}_{\mathcal{H}\left(\sigma_{0}\right)}=\omega_{0, \mathcal{H}\left(\sigma_{0}\right)}\left(B_{0}\right)=\omega_{\tau, \mathcal{H}\left(\sigma_{0}\right)}\left(B_{0}\right),
$$

where $B_{0}$ is the uniformly (w.r.t. $\left.\sigma \in \mathcal{H}\left(\sigma_{0}\right)\right)$ absorbing set in $\mathcal{E}_{0}$.

Proof From Theorem 2.4 and Theorem 3.4, we merely need to prove that the family of processes $\left\{U_{\sigma}(t, \tau)\right\}, \sigma \in \mathcal{H}\left(\sigma_{0}\right)$ satisfy the uniform (w.r.t. $\left.\sigma \in \mathcal{H}\left(\sigma_{0}\right)\right)$ Condition $(C)$ in $\mathcal{E}_{0}$. We assume that $\tilde{\lambda}_{i}, i=1,2, \ldots$ are eigenvalue of operator $A$ in $D(A)$, satisfying

$$
0<\tilde{\lambda}_{1}<\tilde{\lambda}_{2} \leq \cdots \leq \tilde{\lambda}_{j} \leq \cdots, \quad \tilde{\lambda}_{j} \rightarrow \infty, \text { as } j \rightarrow \infty
$$

$\tilde{\omega}_{i}$ denotes eigenvector corresponding to eigenvalue $\tilde{\lambda}_{i}, i=1,2,3, \ldots$, which forms an orthogonal basis in $D(A)$, at the same time they are also a group of canonical basis in $V$ or $H$, and they satisfy

$$
A \tilde{\omega}_{i}=\tilde{\lambda}_{i} \tilde{\omega}_{i}, \quad \forall i \in \mathbb{N} .
$$

Let $H_{m}=\operatorname{span}\left\{\tilde{\omega}_{1}, \tilde{\omega}_{2}, \ldots, \tilde{\omega}_{m}\right\}, P_{m}: H \rightarrow H_{m}$ is an orthogonal projector. For any $\left(u, u_{t}\right) \in$ $\mathcal{E}_{0}$, we write

$$
\left(u, u_{t}\right)=\left(u_{1}, u_{1 t}\right)+\left(u_{2}, u_{2 t}\right)
$$

where $\left(u_{1}, u_{1 t}\right)=\left(P_{m} u, P_{m} u_{t}\right)$.

Choosing $0<\varrho<1$ and $0<\varrho(\alpha-\varrho)<\lambda_{1}$. Taking the scalar product with $v_{2}(t)=u_{2 t}(t)+$ $\varrho u_{2}(t)$ for $(2.8)$ in $H$, we have

$$
\begin{aligned}
& \frac{1}{2} \frac{d}{d t}\left(\left\|v_{2}\right\|^{2}+\left\|u_{2}\right\|_{1}^{2}\right)+\varrho\left\|u_{2}\right\|_{1}^{2}-\varrho(\alpha-\varrho)\left\langle u, v_{2}\right\rangle+(\alpha-\varrho)\left\|v_{2}\right\|^{2} \\
& \quad+k\left\langle u^{+}, v_{2}\right\rangle+\left\langle g(u, t), v_{2}\right\rangle=\left\langle h(t), v_{2}\right\rangle,
\end{aligned}
$$

where

$$
\begin{aligned}
& \left\langle h(t), v_{2}\right\rangle \leq(\alpha-\varrho)\left\|v_{2}\right\|^{2} / 8+2(\alpha-\varrho)^{-1}\left\|\left(I-P_{m}\right) h(t)\right\|^{2}, \\
& \left\langle g(u, t), v_{2}\right\rangle \leq(\alpha-\varrho)\left\|v_{2}\right\|^{2} / 8+2(\alpha-\varrho)^{-1}\left\|\left(I-P_{m}\right) g(u, t)\right\|^{2} .
\end{aligned}
$$

Clearly, we get

$$
\begin{aligned}
& \varrho(\alpha-\varrho)\left\langle u, v_{2}\right\rangle \leq(\alpha-\varrho)\left\|v_{2}\right\|^{2} / 4+(\alpha-\varrho) \varrho^{2}\left\|u_{2}\right\|^{2}, \\
& k\left\langle u^{+}, v_{2}\right\rangle=\frac{1}{2} \frac{d}{d t} k\left\|\left(u_{2}\right)^{+}\right\|^{2}+\varrho k\left\|\left(u_{2}\right)^{+}\right\|^{2} .
\end{aligned}
$$


Combining (3.23)-(3.26), we obtain from (3.22)

$$
\begin{aligned}
\frac{1}{2} \frac{d}{d t} & \left(\left\|v_{2}\right\|^{2}+\left\|u_{2}\right\|_{1}^{2}+k\left\|\left(u_{2}\right)^{+}\right\|^{2}\right)+\varrho\left\|u_{2}\right\|_{1}^{2}+\frac{1}{2}(\alpha-\varrho)\left\|v_{2}\right\|^{2} \\
& +\varrho k\left\|\left(u_{2}\right)^{+}\right\|^{2}-(\alpha-\varrho) \varrho^{2}\left\|u_{2}\right\|^{2} \\
\leq & \frac{1}{2} \frac{d}{d t}\left(\left\|v_{2}\right\|^{2}+\left\|u_{2}\right\|_{1}^{2}+k\left\|\left(u_{2}\right)^{+}\right\|^{2}\right)+\varrho \lambda_{1}^{-1}\left(\lambda_{1}-(\alpha-\varrho) \varrho\right)\left\|u_{2}\right\|_{1}^{2} \\
& +\frac{1}{2}(\alpha-\varrho)\left\|v_{2}\right\|^{2}+\varrho k\left\|\left(u_{2}\right)^{+}\right\|^{2} \\
\leq & 2(\alpha-\varrho)^{-1}\left\|\left(I-P_{m}\right) g(u, t)\right\|^{2}+2(\alpha-\varrho)^{-1}\left\|\left(I-P_{m}\right) h(t)\right\|^{2} \\
\leq & 2 C_{9}(\alpha-\varrho)^{-1}\left\|\left(I-P_{m}\right) g(u, t)\right\|_{\mathcal{M}}^{2}\left(1+\left\|u_{2}\right\|_{1}^{2 \gamma+2}\right) \\
& +2(\alpha-\varrho)^{-1}\left\|\left(I-P_{m}\right) h(t)\right\|^{2} .
\end{aligned}
$$

We define the functional

$$
\mathcal{L}(t)=\frac{1}{2}\left(\left\|v_{2}\right\|^{2}+\left\|u_{2}\right\|_{1}^{2}+k\left\|\left(u_{2}\right)^{+}\right\|^{2}\right)
$$

and we set $\omega=\min \left\{2 \varrho \lambda_{1}^{-1}\left(\lambda_{1}-(\alpha-\varrho) \varrho\right), \alpha-\varrho, 2 \varrho\right\}$, then

$$
\begin{aligned}
\frac{d}{d t} \mathcal{L}(t)+\omega \mathcal{L}(t) \leq & 2 C_{9}(\alpha-\varrho)^{-1}\left\|\left(I-P_{m}\right) g(u, t)\right\|_{\mathcal{M}}^{2}\left(1+\left(\sqrt{2} \mu_{0}\right)^{2 \gamma+2}\right) \\
& +2(\alpha-\varrho)^{-1}\left\|\left(I-P_{m}\right) h(t)\right\|^{2}, \quad \text { for } t \geq t_{0} .
\end{aligned}
$$

By Gronwall's lemma, we obtain

$$
\begin{aligned}
\mathcal{L}(t) \leq & \mathcal{L}\left(t_{0}\right) e^{-\omega\left(t-t_{0}\right)}+\frac{2}{\alpha-\varrho} \int_{t_{0}}^{t} e^{-\omega(t-s)}\left\|\left(I-P_{m}\right) h(s)\right\|^{2} d s \\
& +\frac{2 C_{10}}{\alpha-\varrho} \int_{t_{0}}^{t} e^{-\omega(t-s)}\left\|\left(I-P_{m}\right) g(u, s)\right\|_{\mathcal{M}}^{2} d s, \quad \text { for } t \geq t_{0} .
\end{aligned}
$$

Obviously, there exists a constant $\tilde{C}$, such that

$$
\left\|z_{2}(t)\right\|_{\mathcal{E}_{0}}^{2} \leq \mathcal{L}(t) \leq \tilde{C}\left\|z_{2}(t)\right\|_{\mathcal{E}_{0}}^{2},
$$

so

$$
\begin{aligned}
\left\|z_{2}(t)\right\|_{\mathcal{E}_{0}}^{2} \leq & \tilde{C}\left\|z_{2}\left(t_{0}\right)\right\|_{\mathcal{E}_{0}}^{2} e^{-\omega\left(t-t_{0}\right)} \\
& +\frac{2}{\alpha-\varrho} \int_{t_{0}}^{t} e^{-\omega(t-s)}\left\|\left(I-P_{m}\right) h(s)\right\|^{2} d s \\
& +\frac{2 C_{10}}{\alpha-\varrho} \int_{t_{0}}^{t} e^{-\omega(t-s)}\left\|\left(I-P_{m}\right) g(u, s)\right\|_{\mathcal{M}}^{2} d s
\end{aligned}
$$


Since $g \in L_{c}^{2}\left(\mathbb{R}_{\tau}, \mathcal{M}\right) \subset L_{c^{*}}^{2}\left(\mathbb{R}_{\tau}, \mathcal{M}\right), h \in L_{c^{*}}^{2}\left(\mathbb{R}_{\tau}, H\right)$, from Lemma 2.7, we can know for any $\epsilon_{1}>0$, there exists a constant $m$ large enough such that

$$
\begin{aligned}
& \frac{2}{\alpha-\varrho} \int_{t_{0}}^{t} e^{-\omega(t-s)}\left\|\left(I-P_{m}\right) h(s)\right\|^{2} d s \leq \frac{\epsilon_{1}}{3}, \quad \forall h \in \mathcal{H}\left(h_{0}\right), \\
& \frac{2 C_{10}}{\alpha-\varrho} \int_{t_{0}}^{t} e^{-\omega(t-s)}\left\|\left(I-P_{m}\right) g(u, s)\right\|_{\mathcal{M}}^{2} d s \leq \frac{\epsilon_{1}}{3}, \quad \forall g \in \mathcal{H}\left(g_{0}\right),
\end{aligned}
$$

where $t \geq \tau$.

Let $t_{1}=1 / \omega \ln \left(3 \tilde{C} \mu_{0}^{2} / \epsilon_{1}\right)+t_{0}$, then

$$
\tilde{C}\left\|z_{2}\left(t_{0}\right)\right\|_{\mathcal{E}_{0}}^{2} e^{-\omega\left(t-t_{0}\right)} \leq \frac{\epsilon_{1}}{3}, \quad \forall t \geq t_{1} .
$$

So for every $\sigma \in \mathcal{H}\left(\sigma_{0}\right)$, we get

$$
\left\|z_{2}(t)\right\|_{\mathcal{E}_{0}}^{2} \leq \epsilon_{1}, \quad \forall t \geq t_{1}
$$

where $\left\|z_{2}(t)\right\|_{\mathcal{E}_{0}}^{2}=\frac{1}{2}\left(\left\|u_{2}\right\|_{1}^{2}+\left\|u_{2 t}\right\|^{2}\right)$.

Therefore, the family of processes $U_{\sigma}(t, \tau), \sigma \in \mathcal{H}\left(\sigma_{0}\right)$ satisfy uniformly (w.r.t. $\sigma \in \mathcal{H}\left(\sigma_{0}\right)$ ) Condition $(C)$ in $\mathcal{E}_{0}$. Applying Theorem 2.4, we can obtain the existence of a uniform (w.r.t. $\left.\sigma \in \mathcal{H}\left(\sigma_{0}\right)\right)$ attractor of the family of processes $U_{\sigma}(t, \tau), \sigma \in \mathcal{H}\left(\sigma_{0}\right)$ in $\mathcal{E}_{0}$, which satisfies (3.21).

We thus complete the proof.

So we can draw the conclusion: when the nonlinearity $g(u, t)$ is translation compact and the time-dependent external forces $h(x, t)$ only satisfies Condition $\left(C^{*}\right)$ instead of translation compact, the uniform attractors in $\left(H^{2}(\Omega) \cap H_{0}^{1}(\Omega)\right) \times L_{2}(\Omega)$ exist.

\section{Competing interests}

The authors declare that they have no competing interests.

\section{Authors' contributions}

The authors read and approved the final manuscript.

\section{Acknowledgements}

This work is partly supported by NSFC $(11361053,11201204,11101134,11261053,11101404)$ of China and the Young Teachers Scientific Research Ability Promotion Plan of Northwest Normal University (NWNU-LKQN-11-5).

Received: 5 July 2013 Accepted: 18 March 2014 Published: 28 Mar 2014

\section{References}

1. Lazer, AC, McKenna, PJ: Large-amplitude periodic oscillations in suspension bridges: some new connection with nonlinear analysis. SIAM Rev. 32(4), 537-578 (1990)

2. An, Y, Zhong, C: Periodic solutions of a nonlinear suspension bridge equation with damping and nonconstant load. J. Math. Anal. Appl. 279, 569-579 (2003)

3. Choi, QH, Jung, T: A nonlinear suspension bridge equation with nonconstant load. Nonlinear Anal. 35, 649-668 (1999)

4. Humphreys, LD: Numerical mountain pass solutions of a suspension bridge equation. Nonlinear Anal. TMA 28(11), 1811-1826 (1997)

5. Lazer, AC, McKenna, PJ: Large scale oscillatory behavior in asymmetric systems. Ann. Inst. Henri Poincaré, Anal. Non Linéaire 4, 243-274 (1987)

6. Ma, Q, Zhong, C: Existence of global attractors for the coupled system of suspension bridge equations. J. Math. Anal. Appl. 308, 365-379 (2005)

7. McKenna, PJ, Walter, W: Nonlinear oscillation in a suspension bridge. Nonlinear Anal. 39, 731-743 (2000)

8. Chepyzhov, W, Vishik, Ml: Attractors for Equations of Mathematics and Physics. American Mathematical Society Colloquium Publication. Am. Math. Soc., Providence (2002)

9. Hale, JK: Asymptotic Behavior of Dissipative Systems. Am. Math. Soc., Providence (1988) 
10. Temam, R: Infinite-Dimensional Dynamical Systems in Mechanics and Physics. Springer, New York (1997)

11. Ma, Q, Zhong, C: Existence of global attractors for the suspension bridge equations. J. Sichuan Univ. 43(2), 271-276 (2006)

12. Zhong, C, Ma, Q, Sun, C: Existence of strong solutions and global attractors for the suspension bridge equations. Nonlinear Anal. 67, 442-454 (2007)

13. Ma, S, Zhong, C: The attractors for weakly damped non-autonomous hyperbolic equations with a new class of external forces. Discrete Contin. Dyn. Syst. 18, 53-70 (2007)

14. Lu, S, Wu, H, Zhong, C: Attractors for nonautonomous 2D Navier-Stokes equations with normal external forces. Discrete Contin. Dyn. Syst. 13, 701-719 (2005)

15. Borini, S, Pata, V: Uniform attractors for a strongly damped wave equations with linear memory. Asymptot. Anal. 20 263-277 (1999)

10.1186/1687-2770-2014-75

Cite this article as: Wang et al:: Uniform attractors for non-autonomous suspension bridge-type equations. Boundary Value Problems 2014, 2014:75

Submit your manuscript to a SpringerOpen ${ }^{\odot}$ journal and benefit from:

- Convenient online submission

- Rigorous peer review

- Immediate publication on acceptance

- Open access: articles freely available online

- High visibility within the field

- Retaining the copyright to your article

Submit your next manuscript at $>$ springeropen.com 